\title{
Repeated Superovulation via PMSG/hCG Administration Induces 2-Cys Peroxiredoxins Expression and Overoxidation in the Reproductive Tracts of Female Mice
}

\author{
Sun-Ji Park', Tae-Shin Kim², Jin-Man Kim ${ }^{3}$ Kyu-Tae Chang ${ }^{4}$, Hyun-Shik Lee', and Dong-Seok Lee ${ }^{1, *}$
}

\begin{abstract}
Superovulation induced by exogenous gonadotropin treatment (PMSG/hCG) increases the number of available oocytes in humans and animals. However, Superovulatory PMSG/hCG treatment is known to affect maternal environment, and these effects may result from PMSG/hCG treatment-induced oxidative stress. 2-Cys peroxiredoxins (2-Cys Prxs) act as antioxidant enzymes that protect cells from oxidative stress induced by various exogenous stimuli. Therefore, the objective of this study was to test the hypothesis that repeated PMSG/hCG treatment induces 2-Cys Prx expression and overoxidation in the reproductive tracts of female mice. Immunohistochemistry and western blotting analyses further demonstrated that, after PMSG/hCG treatment, the protein expression levels of 2-Cys Prxs increased most significantly in the ovaries, while that of Prx1 was most affected by PMSG/hCG stimulation in all tissues of the female reproductive tract. Repeated PMSG/hCG treatment eventually leads to 2-Cys Prxs overoxidation in all reproductive organs of female mice, and the abundance of the 2-Cys Prxs-SO $\mathrm{SO}_{23}$ proteins reported here supports the hypothesis that repeated superovulation induces strong oxidative stress and damage to the female reproductive tract. Our data suggest that excessive oxidative stress caused by repeated PMSG/hCG stimulation increases 2-Cys Prxs expression and overoxidation in the female reproductive organs. Intracellular 2-Cys Prx therefore plays an important role in maintaining the reproductive organ environment of female mice upon exogenous gonadotropin treatment.
\end{abstract}

\footnotetext{
${ }^{1}$ School of Life Sciences and Biotechnology, BK21 Plus KNU Creative BioResearch Group, Kyungpook National University, Daegu 702-701, Korea, ${ }^{2}$ Embryology Laboratory, Neway Fertility, 115 East 57th Street Suite 500 New York, NY 10022, USA, ${ }^{3}$ Cancer Research Institute and Department of Pathology, College of Medicine, Chungnam National University, Daejeon 301-747, Korea, ${ }^{4}$ National Primate Research Center, Korea Research Institute of Bioscience and Biotechnology, Daejeon 305-806, Korea

*Correspondence: lee1@knu.ac.kr
}

Received 16 June, 2015; revised 28 July, 2015; accepted 30 July, 2015; published online 19 October, 2015

Keywords: 2-Cys peroxiredoxins, exogenous gonadotropin, female mice, oxidative stress, reproductive organ environment

\section{INTRODUCTION}

Exogenous gonadotropins are used to induce superovulation in humans and animals to increase oocytes and embryo numbers, thereby improving pregnancy success (Practice Committee of American Society for Reproductive Medicine, 2008). Consecutive injections of pregnant mare serum gonadotropin (PMSG) and human chorionic gonadotropin (hCG) is the conventional method used to induce superovulation in rodents (Mukumoto et al., 1995). The PMSG/hCG administration protocol is well established and has been used for mouse superovulation for more than 55 years in many laboratories (Fowler and Edwards, 1957). However, Superovulation in female mice increases the number of unhealthy follicles that ovulate and results in an increase in poor quality oocytes (Ertzeid and Storeng, 1992; Van der Auwera and D'Hooghe, 2001; Walton et al., 1983). Superovulatory treatment causes changes in the unsuitable maternal oviduct and uterine environment which impair implantation and subsequent pregnancy in female mice and rats (Van der Auwera et al., 1999; Walton et al., 1982). Furthermore, repeated ovarian stimulation promotes the apoptosis of ovarian cells and negatively affects ovarian cell function in monkeys (Dong et al., 2014). It seems that these negative effects result from oxidative stress caused by PMSG/hCG-induced superovulation in the female reproductive tract of mice. It has been reported that repeated ovarian stimulation by exogenous gonadotropin induces mitochondrial DNA mutations and oxidative damage to DNA, lipids and proteins in mouse ovaries (Chao et al., 2005). Therefore, it seems that increased oxidative stress by gonadotropin superovulation treatments has detrimental effects on the maternal environment. We believe that antioxidant enzymes preventing repeated PMSG/hCG treatment-induced oxidative stress may play a key role in the female reproductive organ environment.

Peroxiredoxins (Prxs) are the most recently identified family of antioxidant enzymes that reduce $\mathrm{H}_{2} \mathrm{O}_{2}$ via cysteine residue reactivity. The six isoforms of mammalian Prx are classified into three subtypes on the basis of the number and position of cysteine residues that participate in catalysis: four typical 2Cys Prxs (Prx1-4), one atypical 2-Cys Prx (Prx5), and one 1Cys Prx (Prx6) (Chae et al., 2012). Prx1-4 possess two conserved cysteine residues, and in the catalytic cycle of these 2Cys Prxs, the NH2-terminal Cys-SH is first converted by 
peroxide to cysteine sulfenic acid (Cys-SOH). This Cys-SOH then reacts with the conserved $\mathrm{COOH}$-terminal Cys-SH of the other subunit in the homodimer to form a disulfide, which is subsequently reduced by thioredoxin (Trx) (Naranjo-Suarez et al., 2013). As a result of the slow rate of its conversion to disulfide, the sulfenic intermediate is occasionally further overoxidized to cysteine sulfinic acid $\left(\mathrm{Cys}-\mathrm{SO}_{2} \mathrm{H}\right)$ or cysteine sulfonic acid $\left(\mathrm{Cys}-\mathrm{SO}_{3} \mathrm{H}\right)$, leading to inactivation of the peroxidase activity (Rabilloud et al., 2002; Woo et al., 2003). The 2Cys Prxs protect cells from oxidative stress induced by various exogenous stimuli (Rhee et al., 2012) and are essential for preventing neurodegenerative disorders, hemolytic anemia and inflammation from oxidative stress through the elimination of $\mathrm{H}_{2} \mathrm{O}_{2}$ (Kang et al., 2005). The 2-Cys Prxs are also known to be overoxidized in various excessive oxidative stress conditions such as the balloon-injured rat carotids, human atherosclerotic lesions, Pyrazole-induced liver Injury, and aged rat liver (Bae et al., 2012; Kang et al., 2013; Musicco et al., 2009). However, the regulation of 2-Cys Prx expression and overoxidation by repeated superovulatory treatments in the female reproductive tracts of mice has not been demonstrated.

The aim of this study was therefore to assess the effects of exogenous gonadotropin-stimulated superovulation on the reproductive tracts of female mice. We investigated whether repeated PMSG/hCG superovulation treatments induce histomorphological changes in the ovaries, oviducts, and uteri of mice and examined whether intracellular 2-Cys Prx expression and overoxidation (2-Cys $\mathrm{Prx}-\mathrm{SO}_{2 / 3}$ ) are induced by repeated superovulation

\section{MATERIALS AND METHODS}

\section{Animals}

Female ICR mice (10 weeks of age) were purchased from Hyochang Bio-Science (Korea) and maintained in accordance with the institutional guidelines of the Institutional Animal care and Use Committee of the Korea Research Institute of Bioscience and Biotechnology (KRIBB, Korea). All mice were maintained under a 12-h-dark/light cycle, a temperature at $20-22^{\circ} \mathrm{C}$ and a humidity of $50-60 \%$ with continuous supply of chow and water.

\section{Treatment with gonadotropin to induce superovulation}

Female ICR mice were divided into two different treatment groups that were each subjected to induced superovulation. Mice in the first treatment group (PMSG/hCG X1) were injected ip with 5 IU PMSG (Sigma, USA) and then with 5 IU hCG (Sigma) $48 \mathrm{~h}$ later. Mice in the second treatment group (PMSG/hCG X3) were treated with ip injections of 5 IU PMSG and $48 \mathrm{~h}$ later with ip injections of $5 \mathrm{IU}$ hCG. These PMSG/hCG injections were repeated at 1 day intervals to a total of three times. Mice that were not treated with gonadotropin served as a control group (normal). Mice in the normal group were classified as being in the estrus/metestrus stage of the natural estrous cycle by visual observation (Byers et al., 2012) as well as by the assessment of collected tissue samples under a dissecting microscope.

Mice ( $\mathrm{n}=5$ per group) were sacrificed $12 \mathrm{~h}$ after the final PMSG/hCG injection and whole female reproductive organs were collected. Half of the tissue from each mouse was immediately processed for immunohistochemistry, while the ovaries, oviducts, and uteri of the other half were carefully excised and immediately frozen in liquid nitrogen for subsequent protein extraction.

\section{Hematoxylin and eosin staining}

The reproductive tracts of female ICR mice were analyzed by $\mathrm{H} \& \mathrm{E}$ staining in order to examine morphological features using standard methods. The tissues isolated from the mice were fixed with $4 \%$ ormalin (Sigma) overnight, embedded in paraffin, and processed into sections of 3- $\mu \mathrm{m}$ thickness. The sections were deparaffinized and hydrated in brief changes of xylene, alcohol, and water. The sections were then stained with hematoxylin, rinsed, and then stained with eosin. The stained sections were dehydrated in brief changes of water, alcohol, and xylene. After they were mounted, the sections were observed under an Olympus BX51 microscope (Olympus, Japan) under bright field and images were acquired with an Olympus DP 70 camera (Olympus). To obtain an estimate of the total number of follicles and corpus luteum per ovary, the total numbers of primary, preantral, antral ,atretic follicle and corpus luteum were determined in 10 sections from each mouse ovary under a microscope.

\section{Immunohistochemistry}

Tissue were fixed overnight in $4 \%$ neutral buffered formalin, embedded in paraffin, and cut into 3-um thick sections. The sections were deparaffinized and briefly heated for $4 \mathrm{~min}$ in a pressure cooker containing $10 \mathrm{mM}$ citrate buffer $(\mathrm{pH} \mathrm{6.0)}$ for antigen retrieval. All subsequent procedures were conducted at room temperature. The sections were pretreated with $3 \% \mathrm{H}_{2} \mathrm{O}_{2}$ in $0.1 \mathrm{M}$ Tris-buffered saline (TBS; $\mathrm{pH}$ 7.4) for $10 \mathrm{~min}$ to quench endogenous peroxidase activity. The sections were then treated with a protein block solution (Dako, USA) for 20 min and incubated with antibodies directed against Peroxiredoxin 1, Peroxiredoxin 2, Peroxiredoxin 3 (diluted 1:500, ABfrontier) for $30 \mathrm{~min}$ in a humidified chamber. After washing with $0.1 \mathrm{M}$ TBS containing $0.01 \%$ Tween-20 (TBST), the sections were incubated with EnVision anti-rabbit polymer kit (Dako) for $30 \mathrm{~min}$ in a humidified chamber. Peroxidases bound to the antibody complex were visualized by treatment with a 3,3'-diaminobenzidine (DAB) chromogen substrate solution (Dako). The DAB reaction was monitored under a microscope to determine the optimal incubation time and stopped with several washes of $0.1 \mathrm{M}$ TBS. The immunolabeled sections were dehydrated in a graded ethanol series, defatted in xylene, and mounted. Slides were counterstained with hematoxylin before mounting and then evaluated under Olympus BX51 microscope Olympus). The images were acquired with an Olympus DP 70 camera (Olympus).

\section{Western blot analysis}

Lysates of tissue were prepared in ice-cold PRO-PREP buffer (iNtRON Biotechnology Inc., Korea). Proteins were separated on $12 \%$ SDS-polyacrylamide gels and then transferred to $\mathrm{Ni}$ troBind nitrocellulose (Osmonics Inc., USA). The membrane was blocked by blocking buffer and incubated with the following primary antibodies (diluted 1:3000): anti-peroxiredoxin 1, antiperoxiredoxin 2 and anti-peroxiredoxin 3, anti-peroxiredoxin$\mathrm{SO}_{2 / 3}$ (Abfrontier, Korea). Following incubation, membranes were washed and incubated with anti-goat, anti-rabbit, and antimouse IgG horseradish peroxidase (HRP)-conjugated secondary antibody (1:10000, Thermo, Scientific, Korea) for $1 \mathrm{~h}$ at room temperature. After the removal of excess antibodies by washing, specific binding was detected using an ECL kit (Abfrontier). Anti- $\beta$-actin antibody (1:5000, Santa Cruz) was used as a loading control. Band intensities were analyzed using Multi Gauge version 3.0 software (Fuji).

\section{Statistical analysis}

Data are presented as the means \pm SEM of three or more in- 


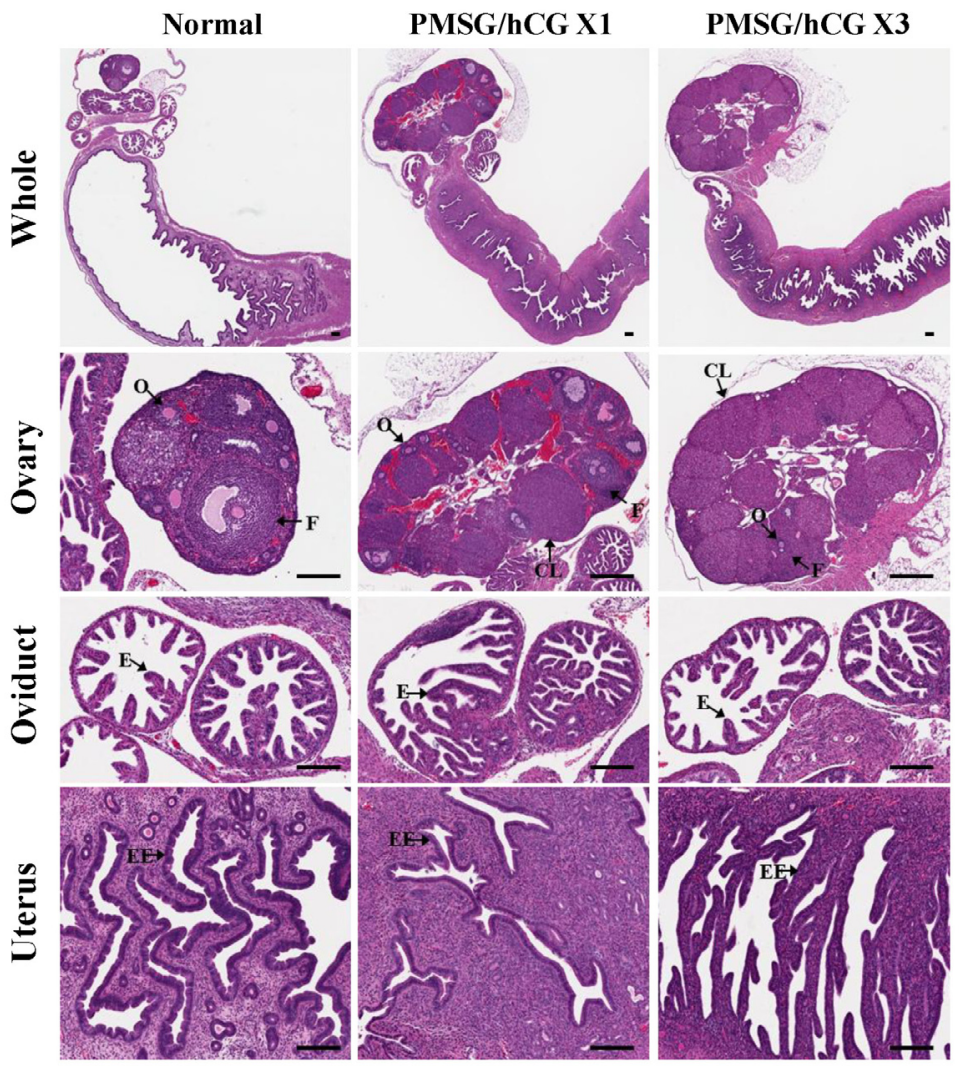

Fig. 1. Histological changes in female reproductive tracts (ovary, oviduct, uterus) of mice following treatment with PMSG/hCG. Whole reproductive tract sections from untreated mice (normal) as well as from mice intraperitoneally (ip) injected with 5 IU PMSG / 5 IU hCG once (PMSG/hCG X1) or three times (PMSG/hCG X3) stained with Hematoxylin and eosin (H\&E). O, oocyte; F, follicle at any developmental stage; CL, corpus luteum; $E$, endometrium; EC, epithelial cells; EE, endometrial epithelial cells; L, lumen. Scale bar $=100 \mu \mathrm{m}$.

Table 1. Ovary size was significantly increased by PMSG/hCG stimulation

\begin{tabular}{ccccccc}
\hline & \multicolumn{2}{c}{ No. of follicles and corpus lutea in each ovary } & & \\
\cline { 2 - 4 } Groups & Follicles & Corpus lutea & Total & & Ovary area $\left(\mathrm{mm}^{2}\right)$ & Endometrial thickness $(\mathrm{mm})$ \\
\hline Normal & $10.5 \pm 1.04$ & $2 \pm 0.41$ & $12.5 \pm 0.96$ & & $1.25 \pm 0.23$ & $0.53 \pm 0.08$ \\
$\mathrm{P} / \mathrm{h} \times 1$ & $9.33 \pm 0.67$ & $7 \pm 0.58$ & $16.67 \pm 0.88^{*}$ & $3.55 \pm 0.73^{*}$ & $0.38 \pm 0.05$ \\
$\mathrm{P} / \mathrm{h} \times 3$ & $6.5 \pm 1.44$ & $11.25 \pm 1.25$ & $17.75 \pm 0.85^{* *}$ & $3.37 \pm 0.61^{*}$ & $0.37 \pm 0.04$ \\
\hline
\end{tabular}

Data are means \pm SEM.

${ }^{*} \mathrm{P}<0.05$; ${ }^{* *} \mathrm{P}<0.01$ when compared with the normal group

dependent experiments. For group comparisons, one-way ANOVA followed by Newman-Keuls multiple comparison tests were performed using the Prism software package version 4.0 for statistical data analysis (GraphPad Software, Inc.). All experiments were repeated independently at least three times. Differences are considered significant at ${ }^{*} p<0.05$; ${ }^{* *}<0.01$; ${ }^{* *}<$ 0.001 .

\section{RESULTS}

Histological changes in female reproductive tract of mice by PMSG/hCG injection

To investigate the effects of repetitive superovulation treatments on the histological morphologies of female reproductive tracts in mice, single $(1 x)$ or repeated $(3 x)$ doses of PMSG/hCG were administered to mice by intraperitoneally (ip) injection. As shown by the results of a histological analysis by hematoxylin and eosin (H\&E) staining (Fig. 1), morphological changes in the ovaries, oviducts, and uteri were induced by PMSG/hCG injection. Most prominently, ovary size was significantly increased by PMSG/hCG stimulation (Table 1). The numbers of follicles and corpus lutea per ovary were also increased by PMSG/hCG treatment. Postovulatory follicles and corpus lutea were observed in the ovaries of the mice subjected to single injections (PMSG/hCG X1 group), but in the case of the mice subjected to repeated injections (PMSG/hCG X3 group), the corpus lutea occupied most of the space in the ovaries. Oviductal morphology of PMSG/hCG-injected mice was not significantly affected compared with that of untreated mice (normal group). In the uterus, PMSG/hCG stimulation resulted in decreases in endometrial thickness and endometrial epithelial cell layers. The uterine lumen surface in mice of the PMSG/hCG X3 group was more jagged and irregular than that in the mice of the other two groups. These findings demonstrate that the morphologies of the superovulated ovaries and uteri were affected by PMSG/hCG administration frequency. 
Altered expression of 2-Cys peroxiredoxins in the PMSG/ hCG-treated female reproductive tract

To investigate whether repeated superovulation affects 2-Cys peroxiredoxins (Prxs) expression and localization in female reproductive organs, the expression of $\operatorname{Prx} 1,2$, and 3 was assessed by immunohistochemistry staining after PMSG/hCG injection. As shown in Fig. 2A, Prx1 expression increased significantly following PMSG/hCG treatment. In the two PMSG/hCG-treated groups (PMSG/hCG X1 and PMSG/hCG $\mathrm{X} 3$ ), highly positive Prx1 staining was observed in almost all regions of the reproductive tract: Prx1 staining was detected in follicles (oocytes, cumulus cells, granulosa cells), corpus lutea (luteal cells), oviductal epithelial cells, and whole endometrium areas of PMSG/hCG-treated mice (Fig. 2D). Prx2 was found to be highly expressed in the corpus lutea in the ovaries of PMSG/hCG X1 and PMSG/hCG X3 mice, whereas Prx2 was found to be only weakly expressed in the oviducts and uteri of the mice of all three groups (Figs. 2B and 2D). Prx3 immunostaining was found to be stronger in the ovaries of PMSG/hCG treated mice than in normal mice and was detected in follicles and the corpus lutea. However, Prx3 expression levels in the oviducts did not differ significantly between the normal and PMSG/hCG-treated groups but were slightly higher in the endometrial epithelial cells of uteri from PMSG/hCG-treated mice than in those from normal mice (Figs. 2C and 2D). Overall, these findings indicate that the expression of the Prx1, Prx2,
$\boldsymbol{A}$

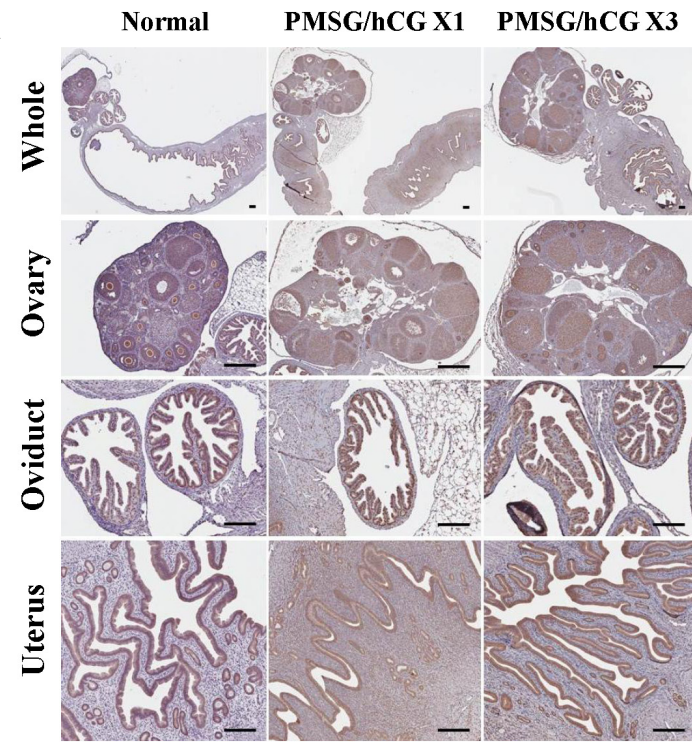

C

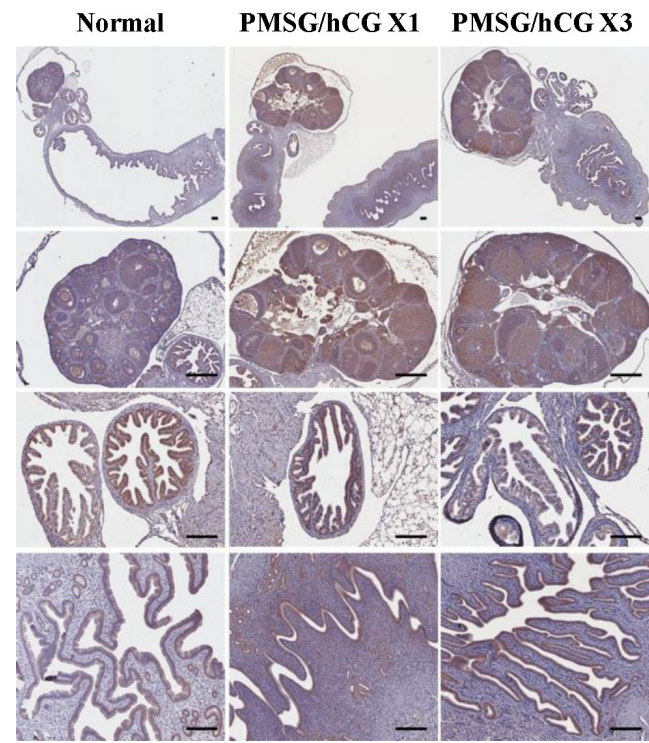

$B$

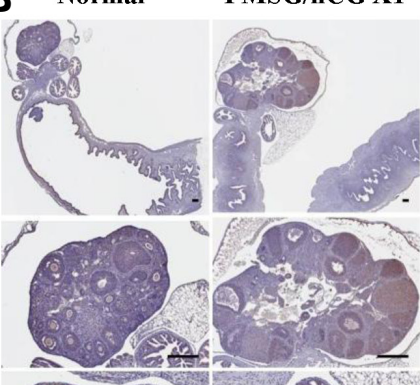

PMSG/hCG X1 PMSG/hCG X3

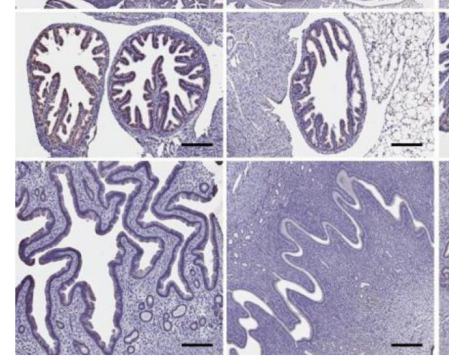

D

Fig. 2. Representative micrographs of immunohistochemistry staining in the ovary, oviduct and uterus of mice. (A) Peroxiredoxin 1-, (B) Peroxiredoxin 2-, and (C) Peroxiredoxin 3-stained sections from untreated mice (normal) as well as from mice ip injected with 5 IU PMSG/5 IU hCG once (PMSG/hCG X1) or three times (PMSG/hCG X3). Scale bar $=100 \mu \mathrm{m}$. (D) The Relative immunoreactivity levels of Prx1-3 in the selected cells of reproductive organ region were assigned -, not detectable; +, weak staining; ++, moderate staining; +++, strong staining. 

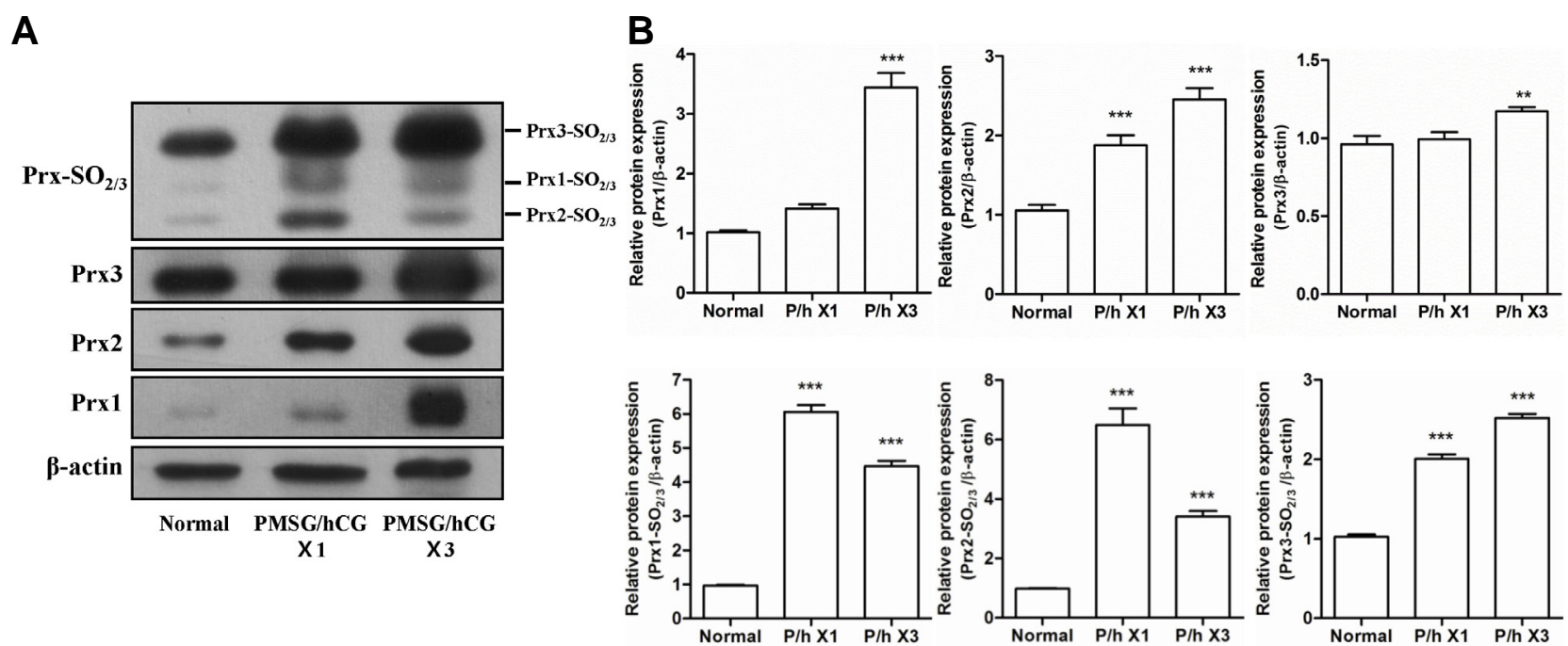

Fig. 3. Expression of the 2-Cys Peroxiredoxins and 2-Cys Peroxiredoxins- $\mathrm{SO}_{2 / 3}$ proteins in the ovaries of mice following stimulation with PMSG/hCG. (A) Western blot analysis of the protein expression levels of $\beta$-actin (loading control), Prx1, Prx2, Prx3, as well as Prx1-, 2-, and 3$\mathrm{SO}_{2 / 3}$ in the ovaries of ICR mice subjected to single $(\times 1)$ or repeated $(\times 3) \mathrm{PMSG} / \mathrm{hCG}$ injections. Ovaries were harvested $12 \mathrm{~h}$ after the final dose of PMSG/hCG. Tissue collection of the untreated mice (normal) was restricted to the estrus/metestrus stage of the natural estrous cycle. (B) Quantification of the relative protein expression levels of Prx1, 2, and 3 as well as Prx1-, 2-, and 3- $\mathrm{SO}_{2 / 3}$ normalized to $\beta$-actin expression. Data in the bar graphs represent means \pm SEM of three independent experiments. ${ }^{* *} p<0.01,{ }^{* \star *} p<0.001$ compared with normal.

and Prx3 proteins in ovaries is significantly increased by PMSG/hCG treatment in mice. After PMSG/hCG stimulation, Prx1 expression is further elevated in oviducts and uteri, whereas significant differences in the expression levels of Prx2 and Prx3 in oviducts and uteri between PMSG/hCG-treated and untreated mice were not detected.

Changes in the protein expression levels of 2-Cys Prxs and 2-Cys Prxs-SO $\mathrm{SO}_{23}$ in the female reproductive tract of PMSG/hCG-treated mice

To compare the effects of PMSG/hCG treatment on the protein expression levels of Prx1, 2, and 3 as well as Prx1-, 2-, and 3$\mathrm{SO}_{2 / 3}$ in each tissue of the female reproductive tract, Western blotting analysis was performed on protein extracted from the ovaries, oviducts, and uteri of mice subjected to PMSG/hCG injection. First, the 2-Cys Prxs and 2-Cys Prxs-SO $\mathrm{SO}_{2 / 3}$ expression levels were assessed in the ovaries collected from mice administered with single $(1 x)$ or repeated $(3 x)$ doses of PMSG/hCG. The protein expression levels of Prx1, Prx2, and Prx3 in the ovaries were found to increase significantly with increased PMSG/hCG injection frequency (Fig. 3A), where, compared with the normal group, Prx1 exhibited the most marked increase in relative expression (3.5-fold) in the PMSG/hCG X3 group, followed by Prx2 (2.5-fold), and then Prx3 (1.5-fold) (Fig. $3 \mathrm{~B}$ ). As shown in Fig. $3 \mathrm{~A}$ and $3 \mathrm{~B}$, the Prxs- $\mathrm{SO}_{2 / 3}$ protein expression levels in the ovaries were also stimulated by PMSG/hCG injection: in the case of Prx1-SO $\mathrm{SO}_{2 / 3}$ and Prx2-SO $\mathrm{SO}_{2 / 3}$, expression in both $\mathrm{PMSG} / \mathrm{hCG}$ groups was markedly increased compared to that in the normal group, where expression in the PMSG/hCG X1 group ( $p<0.001$ compared with normal) was higher than that in the PMSG/hCG X3 group ( $p<0.001$ compared with normal). The protein expression levels of Prx3-SO $\mathrm{SO}_{2 / 3}$ were found to increase with PMSG/hCG injection frequency ( $p$ $<0.001$ compared with normal). Overall, therefore, all 2-Cys Prxs and 2-Cys Prxs-SO $\mathrm{SO}_{2 / 3}$ proteins were shown to be highly expressed in the ovaries of PMSG/hCG-treated mice.
Next, the protein expression levels of 2-Cys Prxs and 2-Cys Prxs- $\mathrm{SO}_{2 / 3}$ in the oviducts were investigated. The protein expression levels of Prx1, 2, and 3 did not differ markedly between the normal and PMSG/hCG-treated groups (Figs. 4A and $4 \mathrm{~B}$ ). The relative protein expression levels of Prx1, Prx2$\mathrm{SO}_{2 / 3}$, and $\mathrm{Prx} 3-\mathrm{SO}_{2 / 3}$ were found to be slightly higher in the PMSG/hCG X1 group ( $p<0.05$ compared with normal) and Prx1-SO $\mathrm{SO}_{2 / 3}$ expression was slightly elevated in the PMSG/hCG $X 3$ group ( $p<0.05$ compared with normal) (Fig. 4B). These data indicate that PMSG/hCG treatment has no marked effect on 2-Cys Prxs and 2-Cys Prxs-SO $\mathrm{SO}_{2 / 3}$ expression in the oviducts of mice.

The protein expression of 2-Cys Prxs and 2-Cys Prxs-SO $\mathrm{SO}_{2 / 3}$ in the uteri of mice was confirmed. As shown in Figs. 5A and 5B, Prx1 expression in uteri of mice in the PMSG/hCG X3 group was found to be 8-fold greater than that in the uteri of normal mice, while the protein levels of Prx2 and Prx3 in the uteri did not change significantly after PMSG/hCG treatment. With increasing frequency of $\mathrm{PMSG} / \mathrm{hCG}$ injection, the expression levels of all $\mathrm{Prx}-\mathrm{SO}_{2 / 3}$ proteins in the mouse uteri were found to increase gradually. These findings reveal that the protein expression levels of Prx1, $\mathrm{Prx} 1-\mathrm{SO}_{2 / 3}, \mathrm{Prx} 2-\mathrm{SO}_{2 / 3}$, and $\mathrm{Prx} 3-\mathrm{SO}_{2 / 3}$ in the uterus were increased by PMSG/hCG stimulation, while PMSG/hCG treatment did not significantly alter the expression levels of Prx2 and Prx3.

Overall, the findings of the Western blot analyses carried out in this study indicate that the protein expression levels of Prx1, 2 , and 3 as well as Prx1-, 2-, and $3-\mathrm{SO}_{2 / 3}$ are elevated in the ovary following single or repeated PMSG/hCG-induced superovulation. In the uterus, the protein expression levels of Prx1 as well as of Prx1-, 2-, and 3-SO $\mathrm{S}_{2 / 3}$ were markedly and gradually increased, respectively, with increasing frequency of PMSG/hCG administration. On the other hand, the relative protein expression levels of Prx1 as well as of Prx1-, 2-, and 3$\mathrm{SO}_{2 / 3}$ in the oviducts were only slight greater than the levels observed in the ovaries and uteri. 
$\boldsymbol{A}$

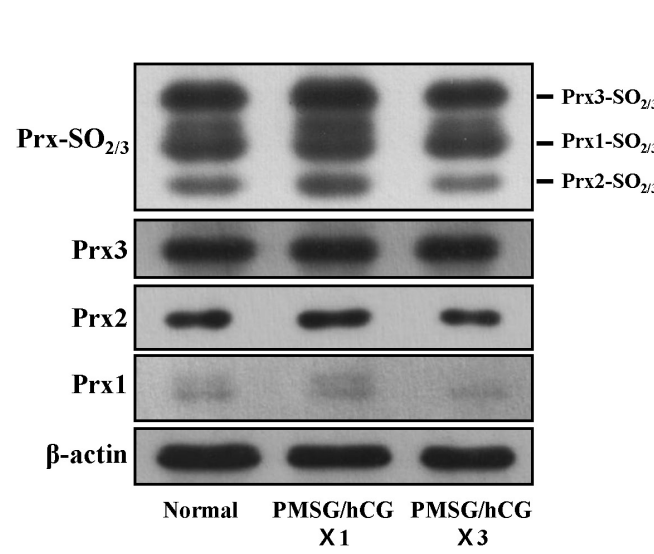

B
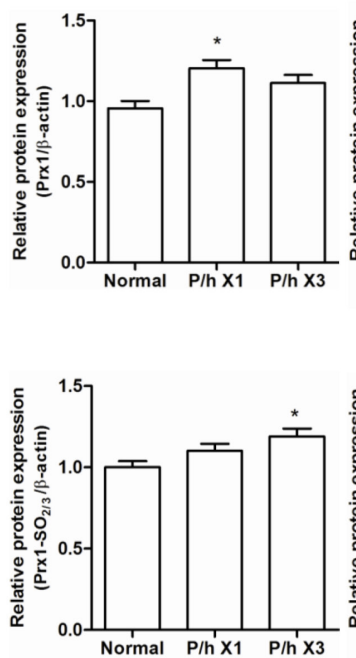
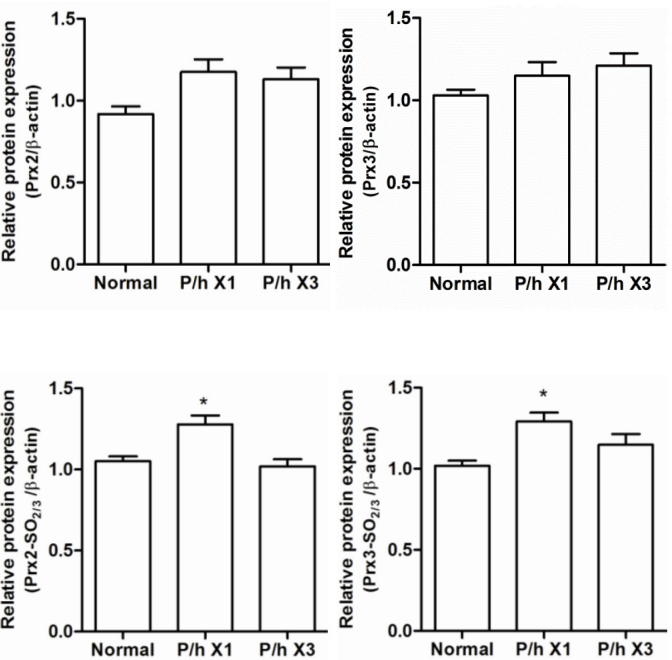

Fig. 4. Expression of the 2-Cys Peroxiredoxins and 2-Cys Peroxiredoxins- $\mathrm{SO}_{2 / 3}$ proteins in the oviducts of mice following stimulation with PMSG/hCG. (A) Western blot analysis of the protein expression levels of $\beta$-actin (loading control), Prx1, Prx2, Prx3, as well as Prx1-, 2-, and 3$\mathrm{SO}_{2 / 3}$ in the oviducts of ICR mice subjected to single $(\times 1)$ or repeated $(\times 3) \mathrm{PMSG} / \mathrm{hCG}$ injections. Oviducts were harvested $12 \mathrm{~h}$ after the final dose of PMSG/hCG. Tissue collection of the untreated mice (normal) was restricted to the estrus/metestrus stage of the natural estrous cycle. (B) Quantification of the relative expression levels of Prx1, 2, and 3 as well as Prx1-, 2-, and 3- $\mathrm{SO}_{2 / 3}$ normalized to $\beta$-actin expression. Data in the bar graphs represent means \pm SEM of three independent experiments. ${ }^{*} p<0.05,{ }^{* *} p<0.01$ compared with normal.

\section{DISCUSSION}

This study demonstrates clearly for the first time that repeated PMSG/hCG superovulation treatment causes histomorphological changes, changes in the expression levels of intracellular 2Cys Prxs (Prx 1-3), as well as overoxidation of 2-Cys Prxs (2Cys Prxs- $\mathrm{SO}_{2 / 3}$ ) in the reproductive tracts of female mice. In this study, it was shown that the histological morphologies of the ovaries and the uteri of mice were affected by repeated PMSG/hCG treatment. A histomorphological assessment by H\&E staining showed the increased sizes of PMSG/hCGtreated ovaries, which can be attributed to a dramatic increase in the number of the superovulated follicles stimulated by gonadotropin. The number of follicles and corpus lutea per ovary was also found to be increased by PMSG/hCG treatment (Fig. 1 ), which suggest that some of the ovarian follicles and corpus lutea observed were unhealthy as a result of the rapid follicle growth following PMSG/hCG injection. It is well known that oocytes of unhealthy follicles in superovulated mice exhibit reduced fertility, increased abnormal blastocyst formation compared with those in naturally ovulating mice (Ertzeid and Storeng, 1992; Van der Auwera and D'Hooghe, 2001; Walton et al., 1983). Moreover, superovulatory treatment causes premature luteal regression, decreases in serum progesterone concentrations that associate with poor embryo development, and embryo implantation (Bainbridge et al., 1998; Stubbings et al., 1986). With increasing numbers of PMSG/hCG injections, the endometrial thickness, endometrial epithelial cell layer of the uteri assessed were noticeably decreased (Fig. 1). Total uterine endometrial thickness and epithelial proliferation at the estrus/metestrus stage are known to be significantly greater than at other stages of the estrous cycle (Wood et al., 2007). The uteri collected from mice $12 \mathrm{~h}$ after the final dose of PMSG/hCG were around ovulation. Although the mice in the PMSG/hCG-treated groups were at the estrus/metestrus stage, the uterine endometrial thickness and epithelial cell layer were decreased in these mice compared to those in the normal group mice at the same stage. Success of embryo implantation depends upon synchronization of the developmental changes in the embryo with those in the maternal endometrium (Modi et al., 2012). During ovarian follicle maturation, the endometrium proliferates, begins to thicken. After ovulation, the endometrium continues to grow to reach a maximum thickness (L.Kierszenbaum, 2002). The blastocyst first attaches to the uterine luminal surface, which is a process directly associated with implantation (Niklaus et al., 2001). In this study, the uterine lumen surfaces of the mice in the PMSG/hCG X3 group were more jagged and rough than those of the other two groups (Fig. 1). These observations suggest that repetitive PMSG/hCG treatment inhibits the development of endometrium thickness, the uterine luminal environment, leading to embryo implantation failure. On the other hand, histomorphological changes were not detected in oviducts after repetitive PMSG/hCG injection. Consequently, these results indicate that repeated gonadotropin stimulation adversely affects the histomorphology and environment of the female reproductive tract in mice, with the exception of the oviduct. It is possible that the ovaries and uterine environments of repeatedly superovulated female mice inhibit blastocyst formation, embryo development, and embryo implantation; however, to investigate this possibility, additional studies would need to be conducted to assess the effects of repeated superovulation on embryo formation, development, and implantation.

Prxs are ubiquitous antioxidant enzymes that act on peroxides in a thioredoxin-dependent manner (Rhee et al., 2012). Typical 2-Cys Prxs (Prx1-4) are highly homologous in terms of protein structure, and in particular they contain two active site cysteine residues. Prx1 and Prx2 are the most abundant antioxidant enzymes in the cytosol, and Prx3 is a major mitochondrial peroxidase. On the other hand, Prx4 appears to reside in 

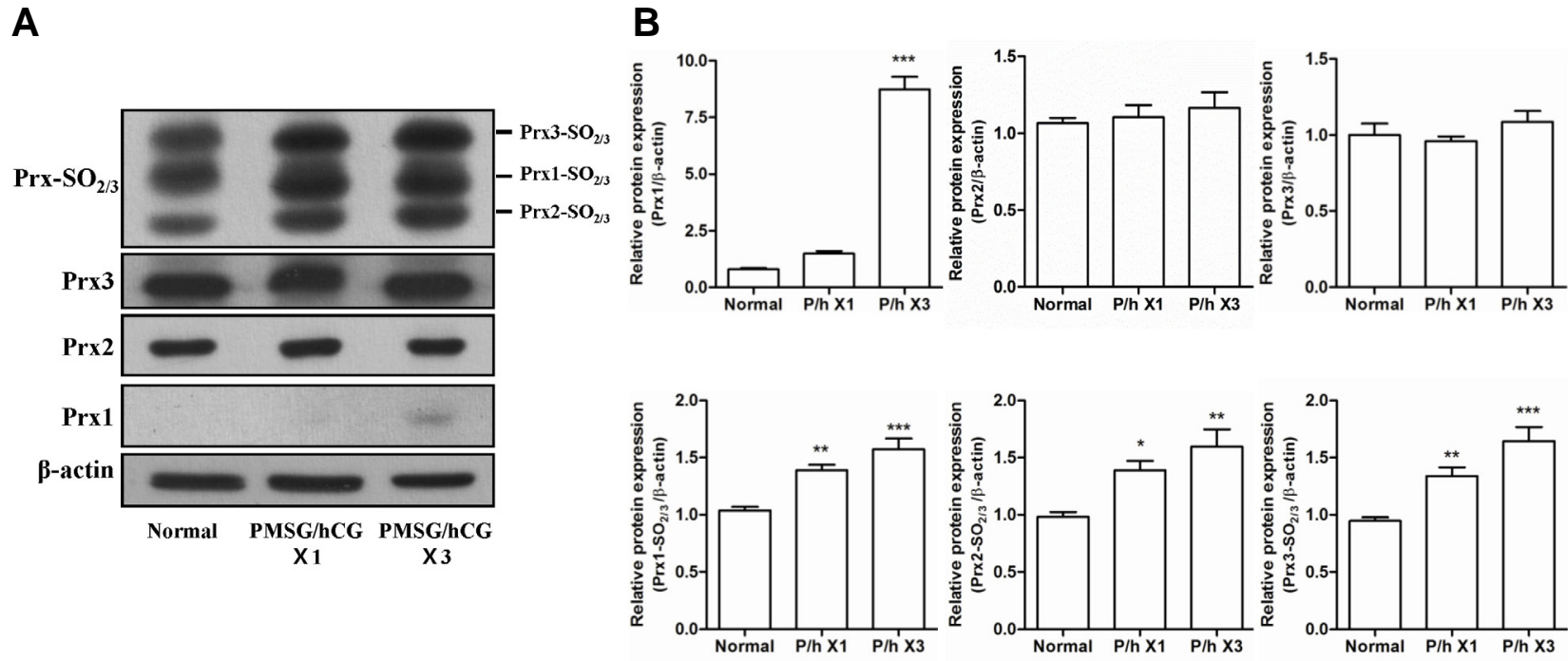

Fig. 5. Expression of the 2-Cys Peroxiredoxins and 2-Cys Peroxiredoxins- $\mathrm{SO}_{2 / 3}$ proteins in the uteri of mice following stimulation with PMSG/hCG. (A) Western blot analysis of the protein expression levels of $\beta$-actin (loading control), Prx1, Prx2, Prx3, as well as Prx1-, 2-, and 3$\mathrm{SO}_{2 / 3}$ in the uteri of ICR mice subjected to single or repeated (3x) PMSG/hCG injections. Uteri were harvested $12 \mathrm{~h}$ after the final dose of PMSG/hCG. Tissue collection of the untreated mice (normal) was restricted to the estrus/metestrus stage of the natural estrous cycle. (B) Quantification of the relative expression levels of Prx1, 2, and 3 as well as Prx-1, 2-, and 3-SO $\mathrm{S}_{2 / 3}$ normalized to $\beta$-actin expression. Data in the bar graphs represent means \pm SEM of three independent experiments. ${ }^{*} p<0.05,{ }^{* *} p<0.01,{ }^{* * *} p<0.001$ compared with normal.

the endoplasmic reticulum (ER) and is secretable due to the presence of a cleavable signal peptide. Therefore, Prx4 has the potential to be present in extracellular fluids, such as serum (Ito et al., 2012). To confirm the presence of intracellular 2-Cys Prxs in the reproductive organs of female mice following $\mathrm{PMSG} / \mathrm{hCG}$ treatment in this study, the expression of Prx1, Prx2, and Prx3 was assessed. Through 2-Cys Prxs immunohistochemistry analysis, we demonstrated that three Prx proteins were highly expressed in the ovaries of mice following PMSG/hCG treatment. The immunostaining levels of Prx1 were higher in PMSG/hCG-stimulated oviducts and uteri than in those from untreated mice, whereas the Prx2 and Prx3 protein levels in the oviducts and uteri of treated mice did not differ significantly from those of mice in the normal group (Fig. 2). A similar result was observed in the expression patterns of Prx1, Prx2, and Prx3 in each female reproductive organ analyzed by western blotting (Figs. 3-5). Among the Prxs, Prx1 expression was most affected by PMSG/hCG stimulation in every tissue of the female reproductive tract. Prx1 expression levels in the tissues of the PMSG/hCG X1 group were slightly elevated even in oviducts in which no morphological changes were detected following PMSG/hCG stimulation (Fig. 4). In contrast, the expression levels of Prx2 and Prx3 were not significantly increased in the female reproductive organs following treatment with PMSG/hCG, with the exception of the ovaries (Figs. 3-5).

The 2-Cys Prxs are overoxidized to cysteine sulfinic acid $\left(\right.$ Cys- $\left.\mathrm{SO}_{2} \mathrm{H}\right)$ or cysteine sulfonic acid $\left(\right.$ Cys- $\left.\mathrm{SO}_{3} \mathrm{H}\right)$, resulting in the inactivation of the peroxidase activity of the enzymes. This overoxidation has been shown to result in irreversible inactivation and has been observed in cells challenged with strong oxidative stress or with long-term treatment with TNF- $\alpha$ (Kang et al., 2005; Rabilloud et al., 2002). Therefore, the overoxidized forms of the 2-Cys Prxs in the female reproductive tracts of repeatedly superovulated mice were assessed in this study. After PMSG/hCG treatment, the expression levels of the 2-Cys
Prxs-SO $\mathrm{SO}_{2 / 3}$ proteins were found to be elevated in all tissues of the female reproductive tract (Figs. 3-5), suggesting that exogenous gonadotropin treatment generates strong oxidative stress thus leading to overoxidation of 2-Cys Prxs. In the ovaries, the expression as well as the overoxidation of the 2-Cys Prxs were significantly increased by PMSG/hCG stimulation (Fig. 3). In the oviducts and uteri, 2-Cys $\mathrm{Prx}-\mathrm{SO}_{2 / 3}$ protein expression was markedly increased after treatment with PMSG/hCG, while Prx2 and Prx3 expression was unaltered (Figs. 4 and 5). These results indicate that the oviducts and the uteri were also damaged by strong oxidative stress generated from repeated PMSG/hCG treatment. To our knowledge, this is the first study investigating the expression levels of 2-Cys Prxs and 2-Cys $\mathrm{Prxs}-\mathrm{SO}_{2 / 3}$ proteins in the ovaries, oviducts, and uteri of mice subjected to single $(\times 1)$ or repeated $(\times 3)$ PMSG/hCG administration.

The overoxidation of 2-Cys Prx, specifically sulfinic acid form, is a reversible reaction, and the reactivation of $\mathrm{Prx}-\mathrm{SO}_{2}$ back to Prx is catalyzed by the enzyme sulfiredoxin (Srx) (Kang et al., 2005). Srx reduces the cysteine sulfinic acid to cysteine, returning it to the catalytic cycle and preventing permanent oxidative inactivation by strong oxidative stress. As a result of the reactivation of overoxidized 2-Cys Prxs, Srx protects cells from apoptosis by maintaining the balance between $\mathrm{H}_{2} \mathrm{O}_{2}$ production and elimination (Jeong et al., 2012). Therefore, Increased levels of inactive 2-Cys Prx by repeated PMSG/hCG superovulation may have induced Srx expression or oxidative stress-mediated pro-apoptotic signaling. The process of 2-Cys Prx recovery by an Srx-dependent process may thus play an important role in regulating oxidative stress-mediated apoptosis in the female reproductive tract of PMSG/hCG-treated mice. In a future study, an attempt will be made to identify whether repeated PMSG/hCG treatment promotes Srx expression, and whether this expression in turn contributes to the inhibition of oxidative stressinduced apoptosis in the female reproductive organs of mice. 
In conclusion, our results indicate that repeated PMSG/hCG stimulation induces histomorphological changes in the ovaries and uteri of female mice. Using immunohistochemistry and western blotting, we demonstrated that, among the reproductive tissues assessed, the expression levels of 2-Cys Prxs after PMSG/hCG treatment are most markedly elevated in the ovaries, and that of the 2-Cys Prxs, Prx1 is most affected by $\mathrm{PMSG} / \mathrm{hCG}$ stimulation in all tissues of the female reproductive tract. Repeated PMSG/hCG treatment was shown to eventually lead to 2-Cys Prxs overoxidation in all reproductive organs of female mice, and the abundance of the 2-Cys Prxs-SO $\mathrm{SO}_{2 / 3}$ proteins reported here indicates that repeated superovulation induces strong oxidative stress and damage to the female reproductive tract. Gonadotropin is used for ovarian stimulation both in animal experiments on embryogenesis as well as in women for the treatment of infertility (Practice Committee of American Society for Reproductive Medicine, 2008); however, our findings suggest that excess gonadotropin stimulation may cause side effects, specifically damage to reproductive function and the maternal environment. This study provides insight that may improve our understanding of the changes that occur in the female reproductive organs following exogenous gonadotropin use.

\section{ACKNOWLEDGMENTS}

This research was supported by a grant (NRF-2014R1A6A 3A01059914, NRF-2014R1A2A1A11054095 and NRF-2015R 1A4A1042271) from the National Research Foundation of Korea funded by the Republic of Korea government, a grant (112020-03-2-SB020) from the Korea Institute of Planning \& Evaluation for Technology in Food, Agriculture, Forestry and Fisheries, and by grants from the KRIBB Research Initiative Program (KGM4611512), Republic of Korea.

\section{REFERENCES}

Bae, S.H., Sung, S.H., Lee, H.E., Kang, H.T., Lee, S.K., Oh, S.Y., Woo, H.A., Kil, I.S., and Rhee, S.G. (2012). Peroxiredoxin III and sulfiredoxin together protect mice from pyrazole-induced oxidative liver injury. Antioxid Redox. Signal. 17, 1351-1361.

Bainbridge, D.R., Deakin, D., and Jabbour, H.N. (1998). Premature luteal regression induced by equine chorionic gonadotropin and estrogen is suppressed by administration of exogenous interferon in red deer (Cervus elaphus). Biol. Reprod. 58, 124129.

Byers, S.L., Wiles, M.V., Dunn, S.L., and Taft, R.A. (2012). Mouse estrous cycle identification tool and images. PLoS One 7, e35538.

Chae, H.Z., Oubrahim, H., Park, J.W., Rhee, S.G., and Chock, P.B. (2012). Protein glutathionylation in the regulation of peroxiredoxins: a family of thiol-specific peroxidases that function as antioxidants, molecular chaperones, and signal modulators. Antioxid Redox. Signal. 16, 506-523.

Chao, H.T., Lee, S.Y., Lee, H.M., Liao, T.L., Wei, Y.H., and Kao, S.H. (2005). Repeated ovarian stimulations induce oxidative damage and mitochondrial DNA mutations in mouse ovaries. Ann. $\mathrm{N} \mathrm{Y}$ Acad. Sci. 1042, 148-156.

Dong, G., Guo, Y., Cao, H., Zhou, T., Zhou, Z., Sha, J., Guo, X., and Zhu, H. (2014). Long-term effects of repeated superovulation on ovarian structure and function in rhesus monkeys. Fertil Steril $102,1452-1457$ e1451.

Ertzeid, G., and Storeng, R. (1992). Adverse effects of gonadotrophin treatment on pre- and postimplantation development in mice. J. Reprod. Fertil 96, 649-655.

Fowler, R.E., and Edwards, R.G. (1957). Induction of superovulation and pregnancy in mature mice by gonadotrophins. J. Endocrinol. $15,374-384$
Ito, $\mathrm{R}$, Takahashi, M., Ihara, $\mathrm{H}$, Tsukamoto, $\mathrm{H}$., Fujii, J., and Ikeda, Y. (2012). Measurement of peroxiredoxin-4 serum levels in rat tissue and its use as a potential marker for hepatic disease. Mol. Med. Rep. 6, 379-384

Jeong, W., Bae, S.H., Toledano, M.B., and Rhee, S.G. (2012). Role of sulfiredoxin as a regulator of peroxiredoxin function and regulation of its expression. Free Radic. Biol. Med. 53, 447-456.

Kang, S.W., Rhee, S.G., Chang, T.S., Jeong, W., and Choi, M.H. (2005). 2-Cys peroxiredoxin function in intracellular signal transduction: therapeutic implications. Trends Mol. Med. 11, 571-578.

Kang, D.H., Lee, D.J., Kim, J., Lee, J.Y., Kim, H.W., Kwon, K., Taylor W.R., Jo, H., and Kang, S.W. (2013). Vascular injury involves the overoxidation of peroxiredoxin type II and is recovered by the peroxiredoxin activity mimetic that induces reendothelialization Circulation 128, 834-844.

L.Kierszenbaum, A. (2002). Histology and Cell biology: an introduction to pathlogy (St. Louis, Missouri, Mosby).

Modi, D.N., Godbole, G., Suman, P., and Gupta, S.K. (2012). Endometrial biology during trophoblast invasion. Front Biosci. (Schol Ed) 4, 1151-1171.

Mukumoto, S., Mori, K., and Ishikawa, H. (1995). Efficient induction of superovulation in adult rats by PMSG and hCG. Exp. Anim. 44 111-118.

Musicco, C., Capelli, V., Pesce, V., Timperio, A.M., Calvani, M., Mosconi, L., Zolla, L., Cantatore, P., and Gadaleta, M.N. (2009). Accumulation of overoxidized Peroxiredoxin III in aged rat liver mitochondria. Biochim. Biophys. Acta 1787, 890-896.

Naranjo-Suarez, S., Carlson, B.A., Tobe, R., Yoo, M.H., Tsuji, P.A., Gladyshev, V.N., and Hatfield, D.L. (2013). Regulation of HIF1alpha activity by overexpression of thioredoxin is independent of thioredoxin reductase status. Mol. Cells 36, 151-157.

Niklaus, A.L., Murphy, C.R., and Lopata, A. (2001). Characteristics of the uterine luminal surface epithelium at preovulatory and preimplantation stages in the marmoset monkey. Anat. Rec. 264 82-92.

Practice Committee of American Society for Reproductive Medicine, B., Alabama. (2008). Gonadotropin preparations: past, present, and future perspectives. Fertil Steril 90, S13-20.

Rabilloud, T., Heller, M., Gasnier, F., Luche, S., Rey, C., Aebersold, R. Benahmed, M., Louisot, P., and Lunardi, J. (2002). Proteomics analysis of cellular response to oxidative stress. Evidence for in vivo overoxidation of peroxiredoxins at their active site. J. Biol. Chem. 277, 19396-19401.

Rhee, S.G., Woo, H.A., Kil, I.S., and Bae, S.H. (2012) Peroxiredoxin functions as a peroxidase and a regulator and sensor of local peroxides. J. Biol. Chem. 287, 4403-4410.

Stubbings, R.B., Bosu, W.T., Barker, C.A., and King, G.J. (1986). Serum progesterone concentrations associated with superovulation and premature corpus luteum failure in dairy goats. Can. J. Vet. Res. 50, 369-373.

Van der Auwera, I., and D'Hooghe, T. (2001). Superovulation of female mice delays embryonic and fetal development. Hum. Reprod. 16, 1237-1243.

Van der Auwera, I., Pijnenborg, R., and Koninckx, P.R. (1999). The influence of in-vitro culture versus stimulated and untreated oviductal environment on mouse embryo development and implantation. Hum. Reprod. 14, 2570-2574.

Walton, E.A., Huntley, S., Kennedy, T.G., and Armstrong, D.T. (1982). Possible causes of implantation failure in superovulated immature rats. Biol. Reprod. 27, 847-852.

Walton, E.A., Evans, G., and Armstrong, D.T. (1983). Ovulation response and fertilization failure in immature rats induced to superovulate. J. Reprod. Fertil 67, 91-96.

Woo, H.A., Chae, H.Z., Hwang, S.C., Yang, K.S., Kang, S.W., Kim, K., and Rhee, S.G. (2003). Reversing the inactivation of peroxiredoxins caused by cysteine sulfinic acid formation. Science 300, 653-656.

Wood, G.A., Fata, J.E., Watson, K.L., and Khokha, R. (2007) Circulating hormones and estrous stage predict cellular and stromal remodeling in murine uterus. Reproduction 133, 10351044. 